\title{
Síndrome de Burnout em professores da rede pública
}

\author{
Gisele Cristine Tenório de Machado Levy UERJ \\ Francisco de Paula Nunes Sobrinho UERJ \\ Carlos Alberto Absalão de Souza UERJ
}

RESUMO

O índice de Burnout foi avaliado em 119 professores da rede pública do ensino fundamental. Os instrumentos de medida consistiram do CBP-R Questionário de Burnout para Professores - R (Moreno; Garrosa; González, 2000) e de um inventário sociodemográfico. Os resultados indicaram que 70,13\% dos participantes apresentavam sintomas de Burnout, sendo que $85 \%$ se sentiam ameaçados em sala de aula. Desses, $44 \%$ cumpriam jornada de trabalho superior a 60 horas semanais e 70\% situavam-se em uma faixa etária inferior a 51 anos. Encontraram-se diferenças significativas para os fatores sentimento de ameaça em sala de aula, jornada de trabalho e idade, quando relacionados ao Burnout. Foram sugeridas alternativas para a humanização do posto de trabalho docente.

PALAVRAS-CHAVE

Síndrome de Burnout, posto de trabalho docente, humanização do posto de trabalho.

\section{Burnout Syndrome in public elementary school teachers}

\begin{abstract}
The Burnout index of 119 Elementary Public Education teachers was assessed. The CBP-R, Teacher Burnout Questionnaire - (Brown, Garrosa and González, 2000) and a socio-demographic questionnaire were used as data collection instruments. The results showed that 70.13 per cent of the participants presented symptoms of Burnout, 85 per cent felt threatened in the classroom, 44 per cent spent more than 60 working hours a week and 70 per cent of them were less than 51 years old. Significant differences were found in the following factors: feeling threatened in the classroom, journey to work and age, when related to Burnout Syndrome. Based on the research results, the authors suggested alternatives aimed at humanizing the teacher workplace.
\end{abstract}

KEYWORDS

Burnout Syndrome, teacher's workplace, humanization of workplaces. 


\section{INTRODUÇÃO}

O objetivo desta pesquisa foi avaliar o índice de Burnout em professores da rede pública municipal, localizada em município de um dos estados da região Sudeste.

A Síndrome de Burnout é considerada uma modalidade de stress ocupacional, que atinge profissionais no desempenho de funções assistenciais. Gil-Monte e Peiró (1997) sustentam a necessidade de se considerar o Maslach Burnout Inventory (MBI), independentemente da sua versão, como um constructo tridimensional, a fim de preservar as características da síndrome. Conceitualmente e para efeitos de avaliação, a Síndrome de Burnout apresenta três fatores distintos: exaustão emocional, despersonalização e baixa realização pessoal (MALAGRIS; CARLOTTO, 2002; CODO, 1999).

- Exaustão emocional: Os trabalhadores têm a sensação de esgotamento e de não poder dar mais de si em termos afetivos. Sentem a energia e os recursos emocionais que dispõem se exaurirem, resultado do intenso contato diário com os problemas de outras pessoas.

- Despersonalização: O trabalhador desenvolve atitudes e sentimentos negativos e de cinismo em relação a clientes e usuários. Há ausência de sensibilidade, manifestada como endurecimento afetivo, "coisificação" das relações interpessoais.

- Baixa realização pessoal: Redução significativa dos sentimentos de competência, relativamente à valorização pessoal que possa ser obti-

da por meio do trabalho cujo objeto são as pessoas.

Os estudiosos do assunto alertam que as condições atuais do magistério concentram, comprovadamente, fatores que contribuem para o stress crônico, podendo evoluir para a Síndrome de Burnout entre os professores, tendo como resultado o absenteísmo e o afastamento desses profissionais de seus postos de trabalho. Nesse cenário, as preocupações com a humanização do posto de trabalho docente começam a despertar o interesse dos ergonomistas.

\section{O QUE APONTAM AS PESQUISAS}

Na medida em que o professor é considerado um trabalhador como outro qualquer, a Síndrome de Burnout se constitui em tópico de interesse para os pesquisadores de áreas diversas do conhecimento (PINDER; TEVEN; TELLES, 2008; LEMOS, 2005; NUNES SOBRINHO, 2008, 2002; LIMA, 2004; BIAZUS; TAVARES, 2000; PARRA, 1992).
Carlotto e Camara (2008) analisaram a produção científica sobre a Síndrome de Burnout no Brasil. Essas pesquisadoras realizaram uma busca bibliográfica nas bases de dados eletrônicas BVS, IndexPsi e Pepsic, em março de 2007. Para tanto, foram utilizadas as palavras-chave Síndrome de Burnout, Burnout e Síndrome do Esgotamento Profissional. Dessa busca, extraíram-se resumos classificados de acordo com as seguintes categorias: revisão teórica, relato de pesquisa e validação de instrumento. Vinte e sete artigos foram identificados, a maioria relatos de pesquisa. Entre as profissões mais investigadas estavam a de professor e profissionais da Saúde, e os resultados foram publicados em revistas de psicologia. Nos anos 2002 e 2005 houve maior produção científica sobre Burnout.

Ao formular a hipótese de que os professores com Síndrome de Burnout são mais suscetíveis de sofrer agressões físicas dos alunos, devido a seu comportamento hostil e punitivo em sala de aula, Pinder (2008) investigou a incidência do Burnout entre esses profissionais da Educação como fator de risco para a violência física no trabalho. Para tanto, foi utilizado um delineamento de pesquisa, considerando-se os casos-controle definidos antes do início do estudo, a partir de um grupo de participantes de uma

\section{Síndrome de Burnout é considerada uma} profissionais no desempenho de funções assistenciais

mesma época histórica (corte). A pesquisa apresentava as mesmas características de um estudo de caso-controle em professores licenciados de Minnesota ( $\mathrm{n}=290$ casos e $\mathrm{n}=867$ controle), para a análise do Burnout. Foi utilizado o Shirom-Melamed Burnout Medida, versão 2, como um fator de risco para a violência física. Ao final do estudo, a pesquisadora confirmou a hipótese de que professores com Burnout correm risco maior de sofrer agressões físicas, concluindo que a violência contra o professor é resultado de seu desgaste físico, emocional e cognitivo.

Teven (2008) investigou as relações entre dois aspectos da personalidade do professor (conscienciosos e neuróticos): o grau de perfeccionismo que os afeta, se são cuidadosos com os alunos, e se apresentam sintomas de Burnout.

Para isso, foi realizado um estudo exploratório, envolvendo 50 docentes da Universidade de Southwestern. Para a coleta de dados foram aplicados os seguintes instrumentos: um questionário de survey, versão reduzida do BIG FIVE measure (SAGAR; GASTIL, 2002), para avaliar os fatores relacionados à personalidade do professor, o Self report 
caring instrument (TEVEN; MCCROSKEY'S, 1997), para verificar se são cuidadosos, e o Maslach Burnout Inventory (MASLACH; JACSON, 1981), para examinar o índice de Burnout. Ao final da pesquisa, foi constatado, dentre outros aspectos, que os docentes que apresentavam um perfil predominantemente perfeccionista encontravam-se mais propensos a desenvolver a Síndrome de Burnout.

Telles (2008) empreendeu um estudo intitulado "Síndrome de Burnout em Agentes Comunitários de Saúde e Estratégias de Enfrentamento", tendo como objetivo constatar a provável ocorrência da Síndrome de Burnout em agentes comunitários de saúde. Optou-se pelo método descritivo, transversal e quantitativo. Foram aplicados questionários - Maslach Burnout Inventory (MBI) e Escala Modos de Enfrentamento de Problemas (EMEP). A amostra foi constituída por 80 ACS (agentes comunitários de saúde) escolhidos aleatoriamente, entre aqueles admitidos há pelo menos dois anos no serviço público municipal.

\section{rofessores com Burnout têm um risco maior de sofrer agressões físicas}

Os resultados mostraram tratar-se de uma amostra predominantemente do sexo feminino $(93,75 \%)$, com filhos (68,75\%), na faixa etária entre 20 e 30 anos (35\%) e com companheiro fixo (60\%). Antes de atuar como ACS, 18,75\% desta amostra trabalharam como auxiliar de serviços gerais, principalmente no âmbito do trabalho doméstico. A renda per capita de $38,75 \%$ é de menos de um salário mínimo, e $53,04 \%$ estão neste trabalho há mais de seis anos. Têm vida sedentária - 66,25\% não praticam atividade física com frequência. O MBI revela um sentimento de deterioração da percepção da própria competência e falta de satisfação desses profissionais com o próprio trabalho, demonstrando estarem emocionalmente esgotados. Verificou-se, ain$\mathrm{da}$, o desenvolvimento incipiente de sentimentos e atitudes negativas e cínicas para com as pessoas por eles atendidas. O modo de enfrentamento mais utilizado são as estratégias focalizadas no problema, seguido pela busca de práticas religiosas/pensamento fantástico. Perceberam-se indícios de sofrimento relacionados ao trabalho característico da Síndrome de Burnout, com o empenho em buscar mecanismos que auxiliem no enfrentamento de problemas relacionados ao exercício profissional. Ao final, a pesquisadora sugere estratégias de acolhimento a esses profissionais para auxiliá-los a lidar com o sofrimento no trabalho.

Quando se trata de stress e Síndrome de Burnout, na abordagem ergonômica da atividade docente, fatores específicos devem ser destacados e, entre eles, os seguintes: a) Carga cognitiva: As tarefas cognitivas do professor se constituem em fatores relevantes na medida em que são requisitadas a todo o momento, na solução de impasses ao longo de sua jornada de trabalho.

b) Lesões, deficiências permanentes ou temporárias: As pesquisas revelam que os professores se queixam de dores lombares e cervicais, laringites e depressões. Esses problemas de saúde estão, provavelmente, associados direta ou indiretamente às condições de trabalho e sobrecarga a que estão expostos esses trabalhadores da Educação (LIMA, 2004; BIAZUS; TAVARES, 2000).

Há indícios de que o distanciamento entre o trabalho pedagógico prescrito (regras, responsabilidades e competências atribuídas ao professor) e o trabalho pedagógico real (a atividade de trabalho) são fatores que contribuem para o stress e a Síndrome de Burnout. Ainda que não disponha de meios para superar o impasse, o professor tenta contornar essa dissociação, sendo que, na maioria das vezes, o fato de não alcançar os objetivos pretendidos acaba por desencadear quadros de ansiedade e depressão (NUNES SOBRINHO, 2008).

Considerando-se como são importantes as condições de trabalho nas quais ocorre a prática da docência, é inevitável o seu impacto sobre a Qualidade de Vida no Trabalho (QVT) do professor. A esse respeito, Biazus (2000) investigou como a implantação de cursos superiores de tecnologia está repercutindo nas condições de trabalho dos professores de uma instituição pública federal de educação tecnológica. Para tanto, o autor coletou informações através de observações e entrevistas informais e semiestruturadas, e da Análise Ergonômica do Trabalho (AET). Ao final da pesquisa, os dados apontaram que para a implantação dos cursos superiores de tecnologia são necessárias estratégias no sentido de se modernizar os laboratórios e fazer adaptações para minimizar consequências físicas e exigências cognitivas nos professores (ABERGO, 2000).

Em relação à sala de aula, local onde o professor permanece a maior parte do tempo e onde se estabelecem as relações de ensino e de aprendizagem, Tavares (2000) empreendeu uma pesquisa abordando os aspectos ergonômicos desse ambiente laboral. O objetivo do autor foi analisar as condições de trabalho docente no Centro Federal de Educação Tecnológica do Rio Grande do Norte - Cefet/ RN, quando da implementação de novas tecnologias didático-pedagógicas. Na pesquisa foi utilizada a metodologia da Análise Ergonômica do Trabalho - AET. Como resultado, ficou claro que, apesar da implantação de novas tecnologias, modificações deverão ser levadas em consideração à luz dos preceitos da ergonomia, tais como a disposição das salas de aula e dos equipamentos, a sua substituição e 
o rearranjo das condições físicas do ambiente, de forma a proporcionar conforto e melhor produtividade no ensino.

Lima (2004) confirma a relevância desse tema, ao destacar estudo realizado por Brestein et al. (2000), em Cincinnati, Estados Unidos. A finalidade dessa pesquisa foi investigar o que poderia, no ambiente de sala de aula, estar causando determinados sintomas nos educadores (coceira, olhos lacrimejando e congestão e sangramento nasal), que trabalhavam em uma escola de $5^{\underline{a}}$ a $8^{\mathrm{a}}$ séries do ensino fundamental. Ao término da pesquisa, os autores concluíram que a presença de certos elementos utilizados como recursos didáticos - como, por exemplo, larvas de insetos para o ensino sobre o ciclo da vida - eram os responsáveis pelos males apresentados pelos professores. fatores que geram desconforto, produzindo, sobre eles, efeitos psicogênicos das cargas físicas.

A necessidade de falar constantemente durante as aulas foi percebida como desconforto para $44 \%$ dos professores; quanto à organização do trabalho, 54,7\% deles mostraramse insatisfeitos com a desproporcionalidade entre a responsabilidade exigida e a remuneração pelo trabalho; $37,2 \%$ afirmaram estar descontentes com o emprego. Há uma distribuição equilibrada de sintomas quanto à idade e ao sexo, sugerindo que o bem-estar psicológico é uma variável interveniente na avaliação da carga psíquica. Os resultados do estudo permitem afirmar que as condições de trabalho desses profissionais são precárias, tornando-os suscetíveis a processos de adoecimento físico e psíquico.

Conforme a literatura revisada sobre o assunto, são múltiplos os fatores que contribuem para o aparecimento da Síndrome de Burnout entre os professores. Nesse cenário, os conhecimentos de interface de domínio do ergonomista conferem a esse profissional habilidades para desenvolver ações preventivas, processos diagnósticos e até a aplicação

O conceito de carga mental ou cognitiva diz respeito ao resultado das inúmeras exigências que mobilizam os processos mentais do professor, ou de qualquer outro profissional, tais como atenção difusa, memória, tomada de decisão e percepção apurada dos fatos durante o contato com os alunos. Nesse ambiente, o professor é levado a tomar decisões múltiplas e diversificadas, em reduzido espaço de tempo, gerando tensão, insegurança e angústia (LIMA et al., 2003).

A pesquisa de Lemos (2005) teve como objetivo caracterizar as cargas psíquicas no trabalho de 318 docentes dos cursos do Centro de Ciências da Saúde da Universidade Federal de Santa Maria/RS e sua influência nos processos de saúde. Essa pesquisa foi iniciada com base em uma análise documental dos afastamentos do trabalho, para tratamento de saúde dos professores. Os procedimentos utilizados compreenderam dois enfoques:

a) Observação das atividades dos professores durante aulas teóricas e práticas, com o intuito de organizar um check-list das condições de trabalho, que serviu de base para a organização do Questionário de Avaliação da Carga Psíquica (Q-CP); e

b) Aplicação do Q-CP. Os resultados apontaram que as condições físicas do ambiente de trabalho (ruídos, estado de conservação dos materiais e equipamentos, exigências posturais) foram citadas pelos professores como de procedimento de intervenção, quando for o caso, no sentido da humanização sistemática das estações de trabalho docente.

Os resultados de pesquisas sobre a Síndrome de Burnout, de modo geral, apontam índices elevados de professores com problemas psicológicos, por acumularem várias funções na mesma atividade, com excessiva carga de responsabilidade, somada à desvalorização do magistério perante a sociedade. Esses resultados indicam também que as doenças evoluem de acordo com o tempo de serviço dos profissionais do magistério.

\section{MÉTODO}

Com base em uma amostra aleatória estratificada, participaram da pesquisa 119 professores da rede pública do ensino fundamental. Esse número representa, aproximadamente, $13 \%$ dos 2.273 professores da rede municipal de ensino que atuam nesse segmento.

A pesquisa foi desenvolvida em cinco escolas de cada um dos polos da rede municipal de ensino. Esses estabelecimentos educacionais são vinculados à Fundação Municipal de Educação e acolhem 27.019 alunos, distribuídos por 82 unidades escolares.

Os instrumentos utilizados para a coleta de dados foram: o CBP-R - um instrumento autoaplicável que avalia a Síndrome de Burnout a partir de fatores antecedentes 
(clima social positivo, preocupações profissionais, stress de papel e condições organizacionais positivas) e de fatores consequentes (realização pessoal, despersonalização e exaustão emocional). Trata-se de um instrumento de medida composto por 66 itens, respondidos em uma escala tipo Likert de 5 pontos, com duração média de aplicação de 30 a 40 minutos, servindo para análise de diferentes fases da Síndrome de Burnout, tais como: Fator I (Stress e Burnout), que compreende itens referentes ao processo de stress e questões relativas ao processo de Burnout e suas dimensões. Distinguem-se nesse fator duas subescalas: Stress de papel e Burnout. No Fator II (Desorganização), há referência às condições nas quais se realiza o trabalho (materiais, recursos de que se dispõe etc.), ao estilo de gestão e ao apoio recebido por parte dos supervisores. possíveis de serem obtidas nos itens discriminantes, conforme mencionados acima, e que se relacionam diretamente ao constructo Burnout. Nesse sentido, a média aritmética foi obtida da seguinte forma:

$$
\begin{aligned}
& \mu=A t / 2 ; \text { onde: } \\
& \mu=\text { média aritmética } \\
& \text { At }=\text { amplitude }
\end{aligned}
$$

Com o objetivo de determinar o ponto de corte na distribuição das pontuações obtidas pelos participantes da pesquisa, relevante para a construção de um diagnóstico diferencial e, no caso, identificar aqueles afetados pela Síndrome de Burnout, foi aplicado o seguinte procedimento:

$$
\begin{aligned}
& \mu=(17+85) / 2 \\
& \mu=51
\end{aligned}
$$

\section{o total dos participantes que sofriam da Síndrome
de Burnout, $86 \%$ se sentiam também ameaçados em}

\section{salas de aula.}

E, por fim, o Fator III (Problemática administrativa), avaliado em função da presença de itens que têm a ver com as preocupações profissionais e o reconhecimento profissional percebido pelos professores: 1 - Preocupações profissionais: alguns exemplos desses itens são "conflitos com a administração" ou "contatos negativos com os pais"; 2 Falta de reconhecimento profissional: "baixo salário", "ausência de serviços de apoio para problemas profissionais". As pontuações altas em cada uma das escalas do questionário indicam mais incidência ou problemática da variável que está sendo analisada (MORENO-JIMENEZ et al., 2000).

Há uma diversidade de formas com que os estudos acadêmicos vêm assumindo a avaliação da Síndrome de Burnout, especialmente no que diz respeito à interpretação do MBI. Os autores estão se referindo aos pontos de corte para estabelecer os níveis alto, moderado e baixo das dimensões, assim como o modo de interpretação do instrumento para definição do diagnóstico (MOREIRA et al.; MAGNAGO; SAKAE; MAGAJEWSKI, 2009).

A pontuação total do questionário é o resultado da soma de todos os pontos das frequências respondidas nos três eixos ou dimensões do constructo (MALLAR; CAPITÃO, 2004). Operacionalmente, o constructo Burnout foi mensurado a partir dos fatores consequentes, discriminados pelos itens $16,17,18,21,22,27,28,36,40$, $42,43,44,46,47,50,51$ e 60 , os quais compõem as dimensões: realização pessoal, despersonalização e exaustão emocional. Para tanto, foi utilizada a média aritmética da amplitude entre as pontuações máxima (85) e mínima (17),
Assim, o ponto de corte tomado como referência para considerar a presença da Síndrome de Burnout nos participantes da pesquisa foi 51 .

\subsection{Planejamento da pesquisa}

Foi utilizado um planejamento entre sujeitos, definido como amostras independentes, considerando-se os seguintes fatores e níveis sobre o constructo lógico Burnout: sentimento de ameaça em sala de aula (professores que se sentem ameaçados e professores que não se sentem ameaçados); jornada de trabalho (mais de 60 horas de trabalho semanais e menos de 60 horas de trabalho); e idade (menor de 51 anos e maior ou igual a 51 anos).

A combinação de fatores especificados e seus níveis permitiu identificar os seguintes grupos de pesquisa:

Grupo 1: Professores que se sentem ameaçados em sala de aula versus professores que não apresentem este sentimento versus Burnout.

Grupo 2: Professores que trabalham 60 horas semanais versus professores que trabalham mais do que 60 horas semanais versus Burnout.

Grupo 3: Professores com idade menor do que 51 anos versus professores com idade maior ou igual a 51 anos versus Burnout.

\subsection{Procedimentos}

O projeto de pesquisa foi submetido à Comissão de Ética da Universidade do Estado do Rio de Janeiro - UERJ.

A aplicação dos instrumentos de coleta de dados ocorreu em cinco escolas da rede, contando com a participação de funcionários da Fundação Municipal de Educação, os quais receberam treinamento específico para a tarefa. Em cada uma dessas escolas foram mantidos os mesmos 
critérios e condições de aplicação dos dois instrumentos: o dia e os horários, e os procedimentos seguidos pelos aplicadores. Esse conjunto de medidas serviu ao pesquisador principal como controle das variáveis intervenientes, que porventura pudessem influenciar nos resultados da pesquisa.

\subsection{Análise dos dados}

Depois de aplicados todos os inventários, os dados coletados foram devidamente processados com o apoio do programa estatístico SPSS 10 (Statiscal Package for Social Science, 2003).

A partir das pontuações obtidas com a aplicação dos instrumentos, inventário sociodemográfico e CBP-R Questionário de Burnout para Professores - R, foram calculadas as médias para Burnout: Condições organizacionais positivas, Preocupações profissionais, Stress de papel e Clima social positivo, considerando-se os seguintes fatores: gênero, professores que trabalham com alunos que têm necessidades educacionais especiais, jornada de trabalho e sentimento de ameaça dos professores em sala de aula.

Em seguida, utilizando-se a estatística paramétrica denominada Teste- $t$ de Student, foi observada diferença significativa entre as médias de Burnout em professores com sentimento de ameaça em sala de aula e aqueles que não se sentiam ameaçados. Em complemento, foi empregada uma Correlação Linear de Pearson com o objetivo de avaliar o grau de associação entre os professores, que expressavam sentimento de ameaça em sala de aula e a idade, levando-se em consideração o Burnout.

\section{RESULTADOS E DISCUSSÃO}

Dentre os 119 professores selecionados para o estudo, $77(64,7 \%)$ responderam aos instrumentos.

Desse total, 54 professores, ou seja, 70,13\% apresentavam sintomas de Burnout. Dentre eles, $85 \%$ sentiam-se ameaçados em sala de aula, $44 \%$ cumpriam uma jornada de trabalho superior a 60 horas semanais e $70 \%$ estavam incluídos na faixa etária inferior a 51 anos. A Figura 1 ilustra a ocorrência da Síndrome de Burnout entre os respondentes.

Como se pode ver, a incidência da Síndrome de Burnout entre os professores que participaram da pesquisa alcançou porcentagens significativas (LEVY; 2006).

\subsection{Sentimento de ameaça em sala de aula}

O fenômeno da violência na sociedade atual vem adquirindo cada vez mais visibilidade. Os resultados obtidos na presente pesquisa evidenciam os efeitos dessa violência sobre a saúde física e psicológica desses profissionais. Do total dos participantes que sofriam da Síndrome de Burnout, $86 \%$ se sentiam também ameaçados em salas de aula, conforme mostrado na Figura 2.

\subsection{A idade do professor $e$ a formação inadequada ou insuficiente}

Por vezes, a idade do professor, somada à sua pouca experiência profissional e à formação inadequada para o atendimento das demandas na atualidade, se transforma em fator que começa a ser observado e registrado pelos pesquisadores. Um dos mais recentes resultados de pesquisa refere-se à idade do professor como elemento determinante para o desenvolvimento da Síndrome de Burnout.

A seguir, a Figura 3 ilustra os respectivos resultados quanto à faixa etária dos participantes. A Figura 3 aponta que $10 \%$ dos professores apresentam idade inferior a 30 anos; $34 \%$ entre 31 e 40 anos; $26 \%$ entre 41 e 50 anos; $25 \%$ entre 51 e 60 anos; e $26 \%$ entre 61 e 70 anos.

Nesse aspecto, a evidência empírica sinaliza que os professores mais jovens, de maneira geral, fazem uso exagerado de controle aversivo em sala de aula, na tentativa de monitorar o comportamento dos alunos a qualquer custo. Todavia, a utilização inapropriada desse controle na manutenção da disciplina desencadeia efeitos colaterais indesejáveis como,

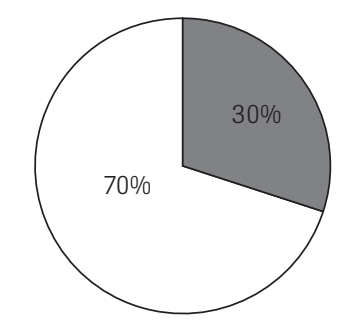

$\square \mathrm{c} /$ Burnout $\square \mathrm{s} /$ Burnout

Figura 1: Distribuição de frequências percentuais de Burnout.

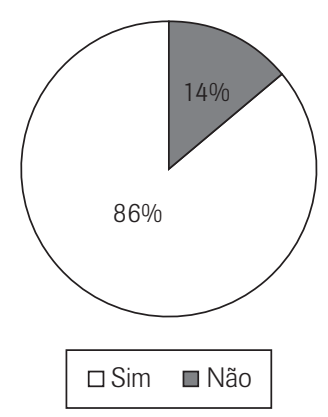

Figura 2: Distribuição de frequências percentuais do sentimento de ameaça em sala de aula. 


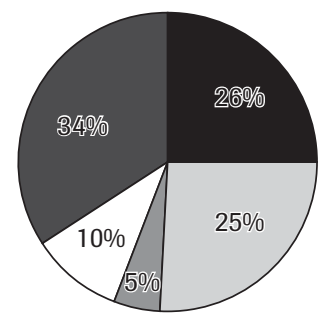

$$
\begin{array}{|ll}
\square \text { Menos de } 30 & \square 51 \text { a } 60 \quad \square 61 \text { a } 70 \\
\square 31 \text { a } 40 & \square 41 \text { a } 50
\end{array}
$$

Figura 3: Distribuição de frequências percentuais relativas às faixas etárias.

por exemplo, sentimentos de revanchismo e exacerbação de condutas agressivas, dentre outras manifestações comportamentais que interferem no processo educacional. Além de menos vulneráveis aos sintomas do Burnout, há indícios de que os professores de mais idade e experiência estão mais capacitados a administrar situações de sala de aula pois utilizam, além dos recursos técnicos, habilidades profissionais adquiridas ao longo do tempo.

Os resultados da presente pesquisa sustentam também que a carga horária de trabalho excessiva contribui para o adoecimento de trabalhadores que atuam em áreas diversas, e o magistério não é exceção, principalmente por estar incluído entre as profissões de risco.

\subsection{Jornada de trabalho}

A jornada de trabalho semanal excessiva é fator que gera incômodo entre os professores. Os baixos salários associados à precariedade do trabalho docente impelem os profissionais a assumirem empregos em várias escolas, na tentativa de complementar seus rendimentos mensais. Trabalhar nessas condições implica mais horas de deslocamentos, maior esforço de adaptação a diferentes ambientes e preparação de atividades escolares distintas, contribuindo para a sobrecarga física e cognitiva do profissional. Como consequência, os dados mostram o aparecimento da Síndrome de Burnout em professores que excedem os limites da jornada de trabalho em mais de 60 horas semanais. Para ilustrar melhor a distribuição dos participantes em relação à jornada de trabalho de mais de 60 horas semanais, veja a Figura 4.

Conforme o gráfico acima, $\mathrm{N}=34$ (44\%) dos professores assumem carga horária de trabalho maior que 60 horas semanais e $\mathrm{N}=43(56 \%)$ carga horária menor que 60 horas semanais.

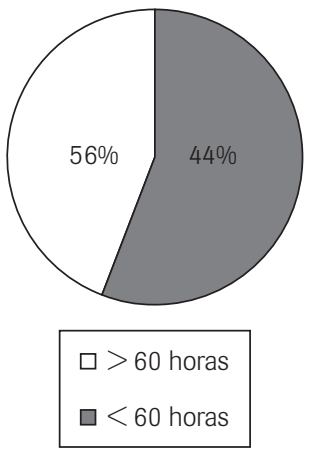

Figura 4: Distribuição de frequências percentuais por jornada de trabalho.

\section{CONCLUSÃO}

Utilizando como referência a literatura e os resultados desta pesquisa, foi possível constatar a presença de fatores decisivos para o desenvolvimento da Síndrome de Burnout entre os professores como, por exemplo, a violência instalada no ambiente escolar, a jornada de trabalho excessiva, os baixos salários, a idade do professor associada à falta de experiência profissional e a formação continuada deficitária para o atendimento das demandas educacionais na atualidade.

Nesse sentido, é válido ressaltar aspectos vinculados à rotina diária do professor, diretamente relacionados à variabilidade do trabalho docente, determinado pelo modo de gestão, políticas educacionais, composição e tamanho das turmas, e infraestrutura material das escolas - carregar material didático, permanecer de pé e em posição inadequada por longos períodos, além do excesso de carga de trabalho.

Os dados apontam também que o professor é um trabalhador sujeito a inúmeros infortúnios, em função das características da própria atividade ocupacional, cada dia mais desumanizada, por conta da desvalorização da profissão. Outros fatores são apontados na literatura como responsáveis pelo grau de esgotamento no trabalho docente, seguidos pelo aparecimento da Síndrome de Burnout.

Assim, os resultados da presente pesquisa sobre o índice da Síndrome de Burnout em professores do ensino fundamental poderão juntar-se a outros para subsidiar políticas públicas no sentido da instalação de serviços de caráter preventivo na rede municipal de Educação. Também possibilitam a elaboração de ações ergonômicas organizacionais de enfrentamento e instalação de programas que auxiliarão o professor no manejo do Burnout, estendendo-se o beneficio à direção das escolas, aos colegas de trabalho e aos alunos.

Os resultados obtidos nesta pesquisa poderão servir para humanizar o posto de trabalho docente, sob os aspectos de saúde, higiene, segurança, bem-estar físico e mental e ambiente ocupacional do professor de sala de aula. Tais 
resultados servirão também para prevenir constantes afastamentos do trabalho, altos índices de absenteísmo, abandono da profissão, problemas no convívio interpessoal com colegas e alunos, baixa produtividade em sala de aula e tantas outras variáveis, que podem ser identificadas como incapacidade do professor de lidar com a Síndrome de Burnout.

\section{Artigo recebido em 20/02/2009 Aprovado para publicação em 15/12/2009}

\section{REFERÊNCIAS}

ASSOCIAÇÃO BRASILEIRA DE ERGONOMIA - ABERGO. [2000]. Disponível em: <http:// www.abergo.org.br $>$.

BIAZUS, M. Condições de trabalho dos professores após a implantação de cursos superiores de tecnologia: estudo de caso em uma Instituição Pública Federal de Educação Tecnológica, a partir da abordagem ergonômica. 2000, 163 f. Dissertação (Mestrado em Engenharia de Produção) - Universidade de Santa Catarina, Florianópolis, 2000.

CARLOTTO, M. Síndrome de Burnout e gênero e os docentes de instituições particulares de ensino. Revista de Psicologia da UnC, v. 1, n. 1, p. 15-23, 2003.

CARLOTTO, M. S.; CAMARA, S. G. Análise da produção científica sobre a Síndrome de Burnout no Brasil. PSICO, Porto Alegre, v. 39, n. 22, p. 152-158, abr.-jun. 2008.

CODO, W. (Coord.) Educação: carinho e trabalho. Petrópolis: Vozes, 1999.

GIL-MONTE, P. R.; PEIRÓ, J. M. Desgaste psíquico em el trabajo: el síndrome de quemarse. Madrid: Síntesis, 1997.

LEMOS, C. J. Cargas psíquicas no trabalho e processos de saúde em professores universitários. 2005. 147 f. Tese (Doutorado em Engenharia de Produção) - Universidade Federal de Santa Catarina, Florianópolis, 2005.

LEVY, G. C. T. M. Avaliar o índice de Burnout em professores da Rede Pública de Ensino fundamental. 2006. 110 f. Dissertação (Mestrado em Educação) - Universidade do Estado do Rio de Janeiro, Rio de Janeiro, 2006.

\section{SOBRE OS AUTORES}

Gisele Cristine Tenório de Machado Levy

Universidade do Estado do Rio de Janeiro - UERJ

Rio de Janeiro, RJ, Brasil

E-mail: giseletlevy@hotmail.com

Francisco de Paula Nunes Sobrinho

Universidade do Estado do Rio de Janeiro.

Rio de Janeiro, RJ, Brasil

E-mail: fnunessobrinho@yahoo.com.br

Carlos Alberto Absalão de Souza

Universidade do Estado do Rio de Janeiro - UERJ

Rio de Janeiro, RJ, Brasil,

E-mail: cabsalao@ig.com.br
LIMA, F. Fatores contribuintes para o afastamento dos professores dos seus postos de trabalho, atuantes em escolas públicas municipais localizadas na Região Sudeste. 2004. 172 f. Dissertação (Mestrado em Educação) Universidade do Estado do Rio de Janeiro, Rio de Janeiro, 2004.

LIMA, F. et al. Humanização do posto de trabalho docente: uma alternativa ergonômica na inclusão educacional. In: NUNES SOBRINHO, F. P. (Org.). Inclusão educacional pesquisas e Interfaces. Rio de Janeiro: Livre Expressão, 2003.

MALAGRIS, L. E. N. Burnout: o profissional em chamas. In: NUNES SOBRINHO, F. P.; NASSALLA, I. (Orgs.). Pedagogia Institucional: fatores humanos nas organizações. Rio de Janeiro: ZIT Editores, 2004. p. 196-213.

MALLAR, S. C.; CAPITÃO C. G. Burnout e hardiness: um estudo de evidência de validade. Psico-USF, v. 9, n. 1, p. 19-29, jun. 2004

MASLACH, C; JACKSON, S. E. The measurement of experienced Burnout. Journal of Ocuppational Behavior, v. 2, p. 99-113, 1981.

MOREIRA, D. S.; MAGNAGO, R. F.; SAKAE, T. M.; MAGAJEWSKI, F. R. L. Prevalência da síndrome de Burnout em trabalhadores de enfermagem de um hospital de grande porte da Região Sul do Brasil. Cadernos de Saúde Pública, Rio de Janeiro, v. 25, n. 7, jul. 2009.

MORENO, B.; GARROSA, E.; JIENEZ, J. L. La evaluación del estrés y el Burnout del profesorado: el CBP-R. Revista de Psicología del Trabajo y de las Organizaciones, v. 16, p. 151-171, 2000.
NUNES SOBRINHO, F.P; NASSARALLA, I. (Orgs.) Pedagogia institucional: fatores humanos nas organizações. Rio de Janeiro: Zit Editores, 2004.

NUNES SOBRINHO, F. P. 0 stress do professor do Ensino Fundamental: o enfoque da ergonomia. In: LIPP, M. L. et al. $O$ stress do professor. 6. ed. Campinas: Papirus, 2008.

PINDER, E. Burnout. do different job stressors predict Burnout in teachers who were physically assaulted versus those who were not? In: ANNUAL MEETING \& EXPOSITION, 136. 2008, San Diego, CA. Anais... San Diego, CA: American Public Health Association - APHA, 2008. Disponível em: $<$ http://www.apha.org $>$.

TAVARES, C. R. G. A Ergonomia e suas contribuições para o processo de ensino aprendizagem: uma análise das salas de aula do CEFET/RN. 2002. 193 f. Dissertação (Mestrado em Engenharia de Produção) - Universidade Federal de Santa Catarina, Florianópolis, 2002.

TELLES, H. Síndrome de Burnout em agentes comunitários de saúde e estratégias de enfrentamento. 2008. 147 f. Dissertação (Mestrado em Enfermagem Psiquiátrica) - Escola de Enfermagem de Ribeirão Preto (EERP), Universidade de São Paulo, São Paulo, 2008.

TEVEN, J. An Exploratory Study of the Relationships among Teacher Temperament, Perfectionist Orientations, and Affective Instruction. In: ANNUAL MEETING OF THE NCA, 94., 2008. San Diego, CA. Anais... San Diego, CA: NCA, 2008. 